\title{
Dificuldades na reabilitação de pacientes amputados devido a tumores
}

Therezinha Rosane Chamlian*

Marcelo Saad**

Danilo Masiero ***

\section{RESUMO}

Os pacientes amputados devido a tumores possuem particularidades, como intercorrências clínicas, que os diferenciam dos amputados por outras causas. Estas particularidades interferem de forma significativa no processo reabilitacional, podendo até inviabilizá-lo. Este levantamento mostra a evolução dos pacientes amputados devido a tumores atendidos no Lar Escola São Francisco. Verificou-se que nenhum dos 6 pacientes atingiu a meta da protetização, apesar de reunirem alguns pré-requisitos favoráveis para reabilitação, como pouca idade e início precoce do treinamento. Isto evidencia que o prognóstico reabilitacional destes pacientes pode ser modificado subitamente em qualquer momento.

\section{UNITERMOS}

Amputados. Tumores. Reabilitação.

\section{SUMMARY}

Amputee patients due to tumors have characterists, like clinical instabilities, that separate them from the amputees due to other causes. These characteristics interfere in a meaningful way with the rehabilitation process, and may even cancel it. This work show the evolution of amputees due to tumors treated at Lar Escola São Francisco. It was verified that no one of the 6 patients met the goal of prosthetics, beside fortunate conditions for rehabilitation as low age and early begining of treatment. This points out that the rehabilitation prognosis of these patients can suddenly be modificated at any time.

\section{KEYWORDS}

Amputee. Tumors. Rehabilitation.

\section{Introdução}

Os problemas de reabilitação encontrados nos pacientes com câncer são multifatoriais e complicados pela evolução inexorável da doença subjacente e seu tratamento. DAHLIN ${ }^{2}$ cita os seguintes fatores complicadores (quadro 1):

\footnotetext{
Disciplina de Fisiatria do Departamento de Ortopedia e Traumatologia da Universidade Federal de São Paulo - Escola Paulista de Medicina Lar Escola São Francisco (DF-DOT-UNIFESP-EPM-LESF)

* Fisiatra, Mestre em Reabilitação pela UNIFESP-EPM

** Fisiatra, Pós-Graduando nível mestrado em Reabilitação pela UNIFESP-EPM

*** Ortopedista e Fisiatra, Chefe da Disciplina de Fisiatria do DOT-UNIFESP-EPM Endereço para correspondência:

Rua dos Açores, 310

CEP: 04032-060 - São Paulo, SP - Brasil
} 
QUADRO 1

Fatores complicadores para a reabilitação de amputação por tumores

- Fraqueza geral pelos efeitos mecânicos causados pelo tumor primário ou suas metástases
- $\quad$ Efeitos tardios da síndrome paraneoplásica
- $\quad$ Problemas nutricionais
- $\quad$ Síndrome do imobilismo
- Problemas psicológicos relacionados ao câncer $(1,2,4)$.

Portanto, o médico fisiatra precisa estabelecer um diagnóstico exato, ter conhecimento da história natural da doença e suas complicações, sendo assim capaz de trabalhar com outros médi$\cos$, como parte da equipe de reabilitação ${ }^{1,4}$.

O câncer metastático para o sistema esquelético é um problema muito mais comum que os tumores ósseos primários. Nos Estados Unidos, os tumores ósseos primários malignos correspondem a $0,5 \%$ de todos os cânceres. O mais comum é o osteosarcoma, que ocorre geralmente na adolescência, envolvendo preferencialmente a extremidade distal do fêmur e a parte proximal do úmero ${ }^{3,5}$.

Antes do desenvolvimento da QT e de procedimentos para poupar os membros (como reposição articular total e imagens radiológicas precisas), a amputação era realizada geralmente em uma articulação proximal ao osso que continha o tumor ${ }^{3,7}$. Os procedimentos de ressecção tumoral com reconstrução esquelética e transferência de músculos são melhor conduzidos em centros terciários de tratamento de câncer, ocasionando menor envolvimento do médico fisiatra nestes casos.

Cirurgias poupando as extremidades estão contra-indicadas quando existe: a-) envolvimento importante de nervos e vasos periféricos; b-) fraturas patológicas; c-) infecção; d-) acometimento muscular extenso; e-) baixa expectativa de vida ${ }^{6}$. Pacientes com amputações devidas a tumores poderiam, teoricamente, utilizar prótese e atingir a meta desejada de independência para marcha. Entretanto, as dificulda$\mathrm{D}=$ direito des comumente encontradas incluem irregularidade ou interrupção do tratamento devido à aplicação de QT ou RT. Isto os diferencia de amputados de outras causas.

Este trabalho tem por objetivo detectar os fatores que interferem no processo de reabilitação de nossos pacientes com diagnóstico de amputação por tumores, e comparar os achados com a literatura.

\section{Material e método}

Foram revisados os prontuários dos pacientes atendidos no Lar Escola São Francisco do período de janeiro de 1993 a junho de 1995. Encontrou-se 86 pacientes amputados de membros inferiores, e destes, 6 amputados por tumor, sendo 4 do sexo masculino $(67 \%)$ e 2 do sexo feminino $(33 \%)$. A idade variou de 3 a 29 anos (mediana de 12 anos). Os seguintes aspectos foram selecionados para análise: tipo de tumor, nível de amputação, procedência, motivo da emigração e ocorrência de QT. Estes aspectos foram confrontados com o grau de sucesso no tratamento reabilitacional. A casuística é apresentada na tabela 1 .

TABELA 1

Casuística, segundo número (A), iniciais (B), sexo (C), idade em anos (D), tipo de tumor $(E)$, nível de amputação $(F)$, procedência $(G)$, motivo da emigração $(H)$, quimioterapia (I)

\begin{tabular}{ccccccccc}
\hline A & B & C & D & E & F & G & $H$ & I \\
\hline 1 & CFS & F & 13 & OS & coxa D & Maranhão & tratamento & sim \\
2 & TAAB & F & 11 & OS & coxa E & São Paulo & - & sim \\
3 & GHSV & M & 12 & OS & coxa E & Bahia & tratamento & sim \\
4 & EJT & M & 19 & OS & perna E & Campinas & tratamento & sim \\
5 & JOVA & M & 29 & OS & coxa D & Maranhão & tratamento & sim \\
6 & FDAL & M & 3 & FS & coxa D & São Paulo & - & não \\
\hline
\end{tabular}

$\mathrm{M}=$ masculino; $\mathrm{F}=$ feminino; $\mathrm{OS}=$ osteossarcoma; $\mathrm{FS}$ = fibrossarcoma; $\mathrm{E}$ = esquerdo; 
Cinco pacientes (83\%) eram amputados acima do joelho, e 1 paciente $(17 \%)$ era amputado abaixo do joelho. Amputação devida a osteossarcoma ocorreu em 5 pacientes (83\%), e por fibrossarcoma em 1 paciente (17\%). Dois pacientes eram procedentes da capital paulista $(33 \%)$ e, dos 4 restantes $(67 \%)$, 1 era do interior de São Paulo e 3 eram de outros Estados. Todos os emigrantes vieram em busca de tratamento especializado oferecido pelo Grupo de Tumores Ósseos do Departamento de Ortopedia e Traumatologia da Universidade Federal de São Paulo - Escola Paulista de Medicina.

O tempo decorrido entre a cirurgia de amputação e o início do tratamento reabilitacional (primeira consulta fisiátrica) foi inferior a 3 meses para 5 pacientes $(83 \%)$ e superior a 1 ano para 1 paciente $(17 \%)$.

\section{Resultados}

Dos 6 casos revistos, 3 pacientes (50\%) tiveram seus tratamentos suspensos devido às faltas nas sessões de fisioterapia, conseqüentes às intercorrências clínicas indesejáveis após QT (\#2, \#3 e \#5). Um paciente (17\%) foi reencaminhado para sua cidade de origem com orientações para prosseguir o tratamento reabilitacional em outro serviço (\#4). Recentemente, recebemos a notícia de que o paciente concluiu QT, recuperou-se fisicamente e foi protetizado com sucesso. Dois pacientes $(33 \%)$ ainda não haviam completado a reabilitação, e estavam em treinamento com suas próteses (\#1 e \#6). Um deles estava começando a ter problemas de frequiência às sessões de fisioterapia devido a efeitos colaterais da QT (\#1).

\section{Discussão}

Foi pequeno o número de casos de amputação por tumor atendidos no período estabelecido ( 2 anos e 6 meses), na revisão de nossos prontuários. Isto pode ser explicado pela tendência à preservação do membro acometido, pelas modernas técnicas cirúrgicas, com indicação de amputação mais precisa e restrita. Assim, os amputados por tumor correspondem a pequena porcentagem das amputações de membros inferiores (na nossa casuística, aproximadamente $8 \%$ dos pacientas amputados).

Nesta amostra, a distribuição do sexo, na proporção de 2 homens para cada mulher, está de acordo com os dados da literatura ${ }^{2}$. O mesmo ocorre para o predomínio do acometimento de adolescentes, por nós observado, e para a in- cidência de osteossarcomas de joelho com maior freqüência ${ }^{2}$.

A maioria dos pacientes $(67 \%)$ era proveniente de fora da capital paulista, muito provavelmente pela escassez de serviços especializados oferecidos fora dos grandes centros, que torna a emigração imperiosa. O índice elevado de abandono do tratamento $(50 \%)$ foi conseqüente às intercorrências clínicas após a QT (febre, náuseas, fraqueza, indisposição, entre outras), que inviabilizavam a prática de exercícios físicos.

O início precoce do tratamento de reabilitação, observado em $83 \%$ dos pacientes, deve-se à boa integração entre a equipe cirúrgica do Grupo de Tumores Ósseos e a Disciplina de Fisiatria, ambos do Departamento de Ortopedia e Traumatologia da Universidade Federal de São Paulo - Escola Paulista de Medicina. Esta abordagem multidisciplinar beneficia o paciente nos aspectos físicos, psíquicos e sócioeconômicos, pois favorece sua reintegração na sociedade na medida em que treina o paciente para o uso da prótese e oferece suporte emocional.

\section{Conclusão}

O prognóstico reabilitacional de pacientes amputados devido a tumores pode ser modificado em qualquer momento, e depende da evolução natural da patologia e das intercorrências clínicas conseqüentes ao tratamento complementar. Em nenhum dos 6 casos revistos houve sucesso na protetização.

\section{Referências bibliográficas}

1. BOCCOLINI, F.: Reabilitação - amputados, amputações, próteses. la. ed., Livraria e Editora Robe. São Paulo, Brasil, 1990

2. DAHLIN, D.C.: Bone fumors - general aspects and data on 6221 cases 3 rd ed., Charles C. Thomas Ed., Springfield, IL. USA, 1978

3. EBSKOV, L.B.: Level of lower limb amputation in relation to etiology - an epidemiological study. Prosth Orth Int 16:163167, 1992

4. KOTTKE, F.J.; STILLWELL, G.K. \& LEHMAN, J.F:: Krusen - tratado de medicina física e reabilitação 3 a. ed., Editora Manole, 1984

5. LEHMANN, J.F.: DeLISA, J.A. \& WARRNE, G. et al.: Cancer rehabilitation - assessment of need, development, and evaluation of a model of care. Arch Phys Med Rehabil 59:410-419, 1978

6. OTIS, J.C.: LANE, J. \& HILLIER, C.D.: Energy consumption in postoperative osteogenic sarcoma patients. In CHAO, E.Y.S. \& INNIS, J.C. (eds.): Design and application of Turner prosthesis for bone and joint reconstrution, pp 385-396. Thieme Stration, NY, USA, 1983

7. SIM, F.H.; BOWMAN, U.U.E. \& CHAO, E.Y.S.: Limb salvage surgery and reconstructive techniques. In SIM. F.H.; THOROFARE. N.J. \& STOCK. C.B.: Diagnosis and treatment of bone tumors - a feam approach, pp 75-105. Slack Inc, Thorofare, NJ. 1983 